\section{Configuración del Turismo en el Ambiente Globalizado. Estudio de casos de Clusters Turísticos}

\section{Geraldo Luciano Toledo Jesús Álvarez Valdés ${ }^{2}$} Álvaro Castroman Pollero

RESUMEN: La estrategia de los agentes del sector turístico está configurán dose competitivamente a partir de estructuras denominadas clusters. En este trabajo se presenta un modelo teórico referencial que analiza las dimensiones de la competitividad de los agentes que pertenecen a un cluster turístico. El alcance de la competitividad de los agentes es proporcional al vector resultante de las estrategias competitivas, las estrategias cooperativas y las de relacionamiento con los turistas. El modelo es desarrollado y examinado a través de la metodología estudio de caso. Los clusters turístico escogidos son: Nordeste de Brasil, Costa Rica y el Sur de Australia.

PALABRAS CLAVES: turismo, estrategias en la globalización, cluster turístico.

ABSTRACT: The strategy of the tourist companies is perfected to reach the competitiveness by the cooperative arrangements in clusters. This paper will present a theoretical reference model to analyze the dimensions of the competitiveness. A tourist cluster reaches your

1. Licenciadoen Administración por laFacultad de Economía, Administración y Contabilidad (FEA) de la Universidad de São Paulo (USP). Prof

2. Doctorando en Turismoen la Faculdad de Economía, Administración y Contabilidade (FEA) de la Universidad de São Paulo (USP). Profesor Adjunto del Centro de Estudios Turísticos de la Universidad de la Habana, Cuba.

E-mail: jesus@usp.br

3. Doctorandoen Turismo en la Facultadde Filosofía,Letras e CienciasSociales (FFLCH) de la Universidad de São Paulo E-mail: alvarocp@usp.br competitive positioning if its companies implemen competitives, cooperatives and relationships strategies. The model is examined by case studies. The tourist clusters of the south of Australia, Costa Rica and northeast of Brazil are appraised with this methodology.

KEYWORDS: tourism, strategyinthe globalitazion, cluster touristic.

\section{Introducción}

La globalización es una referencia constante en las discusiones económicas que están ocurriendo en los últimos años. Envuelve innúmeros y diferentes aspectos, como la apertura de las economías nacionales, la unificación de los mercados y la competencia a escala mundial, que son posibilitados por el nuevo reordenamiento económico, político, tecnológico entre otros.

En este nuevo contexto mundial la estrategia exitosa para una inserción global se estructura a partir de la competitividad, unade las preocupaciones centrales de las empresas y de los gobiernos.

La idea de polo turístico ha llevado al concepto erróneo de relacionar la competitividad turística solamente con las características geográficas, los recursos naturales y turísticos que se poseen en una determinada región. Se sabe que en el sector turístico este concepto de polo ha comenzado actualmente a ser cuestionado por el éxito de los clusters en otros sectores de la economía como los de la informática, el automovilístico, el químico, entre otros, y los resultados concretos de clusters turísticos en países como EUA, Canadá, Australia, México y Costa Rica.

Las principales características y resultados de las configuraciones en clusters son analizados en este trabajo a través de teorías específicas del sector turístico y de otros sectores más desarrollados en niveles de organización y competitividad. Los objetivos de este trabajo son:

- destacar las relaciones básicas a desarrollar en la configuración de los clusters turísticos de forma tal de asegurar la competitividad sustentable de las empresas turísticas de una región;

- contribuir con un modelo de integración de los principales factores que deben ser considerados para el desarrollo de la ventaja competitivasustentable de un cluster turístico en la actual fase de la globalización.

Se presenta una metodología para la formación de un cluster turístico que se ejemplifica mediante el estudiode casos en sus diferentes estadios de desarrollo. Los clusters turísticos analizados son: Sur de Australia (Estadio de Sustentabilidad); ecológico de Costa Rica (Estadio de Desarrollo); el Nordeste de Brasil (Estadio de Iniciación). 
Nuevas Configuraciones Competitivas en la Actual Fase de la Globalización: clusters

Se sabe que los países no pueden ser competitivos en todos los sectores de la actividad económica. Según Porter (1998), la variable tiempo-espacio, en la actual fase de la globalización, ha desconfigurado la mayoría de las fuentes de ventajas comparativas tradicionales. El autor destaca que la búsqueda de competitividad actual ha tenido como resultado configurar el mapa económico del mundo con una nueva estructuración espacial que se denomina cluster. Lo define como concentraciones geográficas de empresas y instituciones interconectadas masalláde la competencia entre ellas. Sus características principales son:

- ser un conglomerado multisectorial de empresas e organizaciones integradas sinergicamente (Cravens, Piercy; 1994; Slater, Narver, 1995; Hunt, Morgan, 1995);

- produciruna masa críticaque agregamayorvalora su oferta, mediantela actividad conjunta en los portafolios de productos de todas las empresas organizadas según esta configuración (Dull, Mohn, Noren, 1995);

- estar localizado en un local geográfico específico con una fuente especial para la competitividad (Porter, 1998);

- estar por encima de los intereses nacionales y regionales (Cravens, Piercy, 1994; Slater, Narver,1995; Hunt, Morgan 1995);

- ser típicos y diferenciados aunque no únicos (Porter, 1998);

- alcanzar las ventajas competitivas por sus conocimientos y relaciones trabajadas en el ámbito local mejor que sus competidores globales (Cravens, Piercy, 1994; Slater, Narver, 1995; Hunt, Morgan 1995);

- estar integradas sus empresas verticalmente (incluyendo canales de distribución y clientes) y en la horizontal (integración con productos suplementarios, industria de tecnologías anexas y abastecimientos comunes) (Porter 1998; Dull, Mohn, Noren, 1995).

Un cluster presenta características intrínsecas de la actual fase de la globalización, pues integra, con un objetivo común, las diversas instituciones intersectoriales que surgen de la nueva relación entre entidades: públicas, privadas, mixtas, gubernamentales, no gubernamentales, bilaterales, universidades, asociaciones comerciales de clase, órganos de investigación, entre otras.

Según Dull, Mohn, Noren (1995), constituye una forma alternativa de organizar la cadena de valor a través de redes de alianzas y sociedades entre las empresas de forma tal de aumentar y facilitar la flexibilidad para superar la rígida competencia tradicional.

Como destacan Cravens, Piercy (1994), el fenómeno de formación de cluster atrae naturalmente la presencia de empresas correlacionadas entre sí para un país o región, para alcanzar la ventaja competitiva de forma conjunta. La presencia de industrias internacionalmente competitivas tiene un efecto multiplicador que proporciona clientes sofisticados, interna y externamente, y estimula los servicios relacionados. Para Porter (1998) este tipo de configuración alcanza su éxito por la cooperación entre todossus integrantes, cuando se consolida una historia de éxito de renombre mundial, que pasa a tener el efecto de atracción competitiva sclectiva. Se favorece por cl propio interés de coordinación de las empresas actuantes, la entrada de las empresas realmente más competitivas, eliminando las barreras tradicionales. Con el arribo de estos nuevos competidores, empresas complementarias y demás factores se produce un efecto multiplicador de eficiencia y competitividad.

\section{Sistema Interfuncional Interrelacionado de la Competitividad en Destinos turísticos (SIIC)}

La OMT (1998) considera que el sector de turismo actual debe considerar los principales cambios ocurridos para planificar su competitividad internacional.

\section{Cambios en las Características de los Clientes}

El turista actual presenta un comportamiento, como consumidor, diferente al pasado, por el aumento de su calificación y experiencia. Como destaca Beni (2000), buscará más la calidad que la forma. Debido a su calificación y la gran oferta competitiva existente en todo el mundo, no consumirá cualquier producto turístico existente. El turista posee mayor poder de decisión. Conoce de la relación preciocalidad de los productos, a partir del valor que le agrega en la satisfacción de sus expectativas. Toledo et al. (2001) señalan que los conceptos de satisfacción y fidelización al turista, aunque son muy mencionados, noestán totalmenteinteriorizados en los objetivos estratégicos y tácticos de gestión de las empresas y en muchos casos se continúa utilizando erradamente la estrategia de crecimiento de la oferta como factor generador de la demanda, principalmente en regiones con destacados atractivos turísticos. A partir de estos cambios se ha comprobado que el consumidor turístico percibe como mejor destino, aquel que le proporciona una oferta integrada de productos/servicios y no una oferta dispersa de hoteles, playas, restaurantes, museos y otros, que normalmente son brindados por prestadores diferentes y no relacionados.

\section{Cambios en el Sector}

Valls (1996) señala que el sector inicialmente se estructuró a partir de la concentración simple y aleatoria de empresas en torno de algún atractivo turístico con ventaja comparativa al nivel de la región (polo turístico). Gutiérrez, Bordas 
(1993) destacan que actualmente la competencia entre los mercados turísticos internacionales serealiza cada vez en clusters turísticos orientados estratégicamente a segmentos específicos de turistas y su fidelización.

\section{Consideración de la Sustentabilidad del Sector}

Manning (1993) destaca que es vital que la planificación estratégica de cada empresa tenga por referencia las líneas generales del plan estratégico del destino turístico, pudiéndose así estructurar una base de convergencia de los intereses públicos y privados con criterio de sustentabilidad. También considera como, a partir de los resultados de las diversas comisiones mundiales sobre el medio ambiente y el desarrollo, se ha comenzado la divulgación del "desarrollo sustentable" como un objetivo para toda la sociedad humana.

Manning (1993) relaciona la competitividad a largo plazo del destino urístico con la sustentabilidad de una serie de atracciones como son: ambiente natural-artificial,identidad, cultura, historia, y del valorcivilizatorioquela comunidad le pueda agregar al espacio regional.

\section{Planificación Estratégica en el Sector de Turismo}

Mintzberg, Quinn (1998) definen la dimensión más importante de la planificación estrategia como la perspectiva de que los integrantes, que forman el destino, visualizan como el mundo funciona en la globalización. Una estrategia de desarrollo de la competitividad del destino turístico combinará los intereses empresariales de corto y largo plazo con los criterios de sustentabilidad de la región. Lo que es primordial, es la formación de estrategias intersectoriales, en que las acciones pasen a ser ejercidas con una base colectiva y articulada para satisfacer al turista.

El sistema de turismo, destaca Beni (2000), debe ser configurado a partir de relacionamiento con el cliente (turista), que es el centro del funcionamiento de cluster. Para poder alcanzar la fidelización del cliente, su gestión debe ser competitiva, cooperativa y proactiva, ejercida por todos sus integrantes.

A partir de Porter (1996), que define los cuatro atributos que promueven (o impiden) la creación de la ventaja competitiva nacional, se propone, en este estudio un modelo teórico referencial que oriente metodológicamente los análisis y estudios sobre la integración de los elementos del sistema turístico y sus interrelaciones en un cluster. ElSIIC quese propone, estáformadoporcincoelementos y sus interrelaciones en el sistema turístico. Los dos primeros, masa crítica y naturaleza de la demanda, tienen las características de crear condiciones básicas para el desarrollo del cluster. Los tres elementos restantes del modelo son los que mejoran la ventaja competitiva del cluster y son: estrategia competitiva, estrategia cooperativa y estrategia de relacionamiento con el cliente. Estos elementos se interrelacionan para crear la ventaja competitiva como muestra la Figura 1.

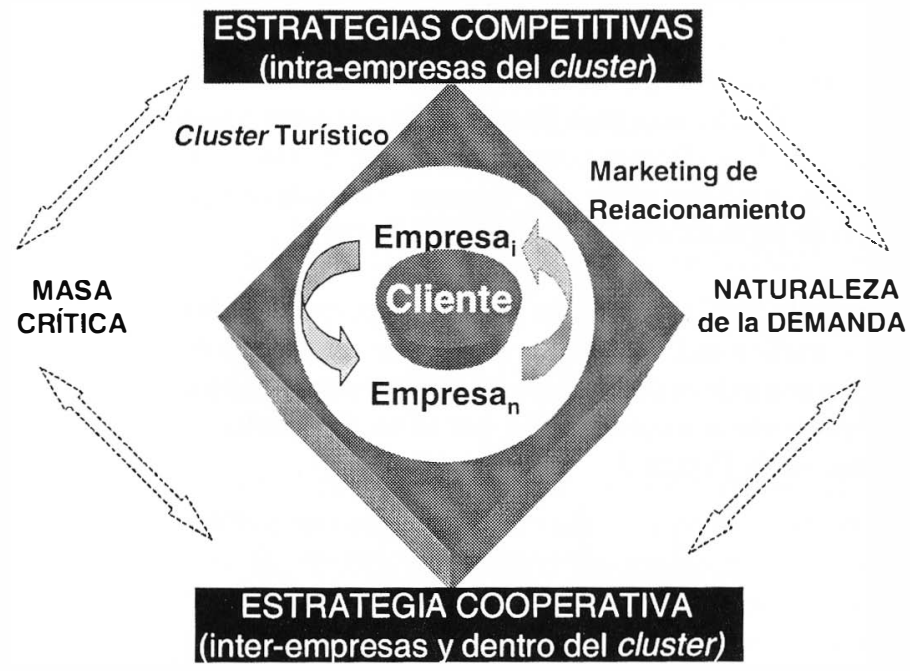

Fuente: los autores

\section{FIGURA 1 - MODELO DEL SISTEMA INTERFUNCIONAL INTEGRADO DE LA COMPETITIVIDAD EN DESTINOS TURISTICOS (SIIC)}

- masa crítica: es el conjunto de los factores básicos necesarios para el desarrollo del destino turístico. Abarca la integración de los recursos turísticos; cultura, inversiones, infraestructura, folklore, salud, superestructura, seguridad entre otros;

- naturaleza de la demanda: se destacan principalmente tres factores que determinan la demanda: a) naturaleza, deseos y expectativas del consumidor turístico; b) potencial de la demanda; c) padrones de calidad o sofisticación de los segmentos abordados;

- estrategia competitiva: según Porter (1996) una rivalidad interna vigorosa, sin restricciones y en igualdad de condiciones para competir, crea un ambiente fértil en el cual las empresas del cluster pueden crecer competitivamente. Este tipo de competición exige estrategias actualizadas y la constante introducción de innovaciones para satisfacer mejor las necesidades del cliente. Un grupo de rivales internos fuertes en el cluster constituye un ingrediente esencial para el suceso competitivo en este tipo de configuraciones. El suceso internacional del cluster exige un ambiente local, que coloque las empresas en padrones internacionales de calidad de servicios y que facilite, en lugar de inhibir, estos padrones de competitividad;

- estrategia cooperativa: estrategia básica del cluster, tienecomo propiedad principal 
el estrechamiento de las relaciones entre las organizaciones regionales, para crear una red de sinergias en busca de objetivos comunes como la satisfacción del cliente y de la sociedad local. Su objetivo, a escala mundial, es el desarrollo de la ventaja competitiva respecto a otros clusters:

- estrategia de relacionamiento con el cliente: en los modelos de gestión actuales, los relacionamientos con los clientes son altamente jerarquizados al igual que entre empresas. Los clientes con sus inputs (necesidades, deseos y expectativas), optimizan la configuración del sistema, retroalimentándolo con el proceso simultáneo de producción-consumo-evaluación

El modelo SIIC sugiere que la diferencia real del cluster turístico con la del polo turístico tradicional, está en que además de considerar los elementos de partida (masa crítica y naturaleza de la demanda), imprescindibles para desenvolver al polo, el cluster crea su ventaja competitiva por el vector resultante de las tres estrategias representadas en la Figura 2.

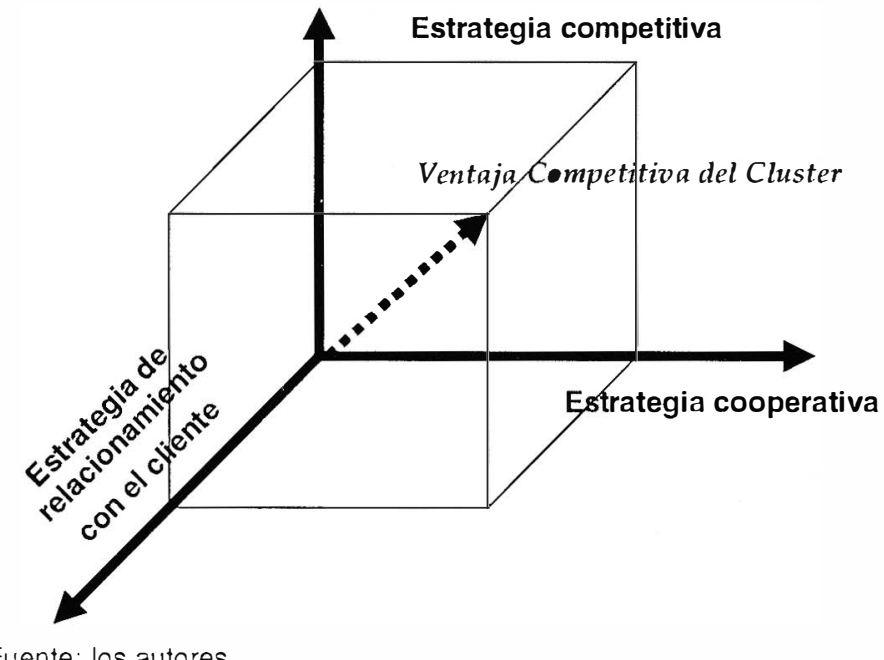

Fuente: los autores

FIGURA 2 - COMPETITIVIDAD DE LAS EMPRESAS DE UN CLUSTER TURISTICO

El cluster desarrolla una forma de configuración de la producción espacial, en tres direcciones:

a) integración vertical del agente en la busca de su ventaja competitiva;

b) integración horizontal entre los agentes, en la busca de la cooperación y articulación para obtener un desempeño superior colectivo ante otros clusters;

c) integración en el valor a ser agregado al cliente mediante un relacionamiento más efectivo.
Para fundamentar esta propuesta, se ha resumido los siguientes conceptos de Porter (1998), Achrol (1991); Webster (1992); Manning (1993), McKenna (1993); Hunt, Morgan (1995), como muestra lo Cuadro 1.

\section{Estudio de Casos de Clusters Turísticos}

A partir del análisis de los planos estratégicos de los clusters estudiados, la metodología del modelo SIIC destaca las siguientes características de los casos escogidos:

\section{Caso: Sur de Australia}

Smith et al. (1999), afirma que la formación y desarrollo de este cluster turístico comenzó con un proceso que buscó encontrar las principales tendencias del sector, sus puntos fuertes, las necesidades para el desarrollo, con una participación amplia de los líderes de las iniciativas públicas, privadas y no gubernamentales regionales. La viabilidad del proyecto se determinó por el potencial estimado para asegurar la tasa de crecimiento de las visitas internacionales del 8\% anual, (19972005), la capacidad para generar $\$ 700$ millones anuales y más de 12.800 empleos adicionales.

La identificación en la región de las potencialidades para la organización en cluster turístico, fue ejecutada por un comité promotor, constituidopor representantes de las tres iniciativas. Este comité promotor comenzó por convocar a todos los stakeholders a participar del proceso de formación del cluster, utilizando la siguiente metodología:

a) distribución a todos los interesados de un documento base que contiene la pauta y el posible modelo teórico directriz (background paper);

b) elaboración y explicación de un mapa de todas las organizaciones del cluster

c) caracterización del sistema turístico de la región;

d) formación de equipos de trabajo por temas-problemas;

e) elaboración de proyectos específicos: inversiones en infraestructura; marketing para el cluster; colaboración en I+D; desarrollo de la marca del cluster; desarrollo del producto turístico.

Smith et al. (1999) presenta metodologías y datos del plano estratégico del cluster en que demuestran que para alcanzar el éxito, como fue planeado, están fuertemente involucradas instituciones públicas, privadas y no gubernamentales en un mismo plano de incentivos y objetivos. La metodología del modelo SIIC, confirma porque la OMT (1998) da como ejemplo de turismo exitoso a Australia, donde es articulada de manera eficiente la actuación del cluster en las tres direcciones estratégicas principales (competitiva, cooperativa y de relacionamiento con el cliente) para buscar su ventaja competitiva en nivel mundial. 
CUADRO 1 - CARACTERISTICAS DE LAS ESTRATEGIAS DE LAS EMPRESAS PARA ALCANZAR LA VENTAJA COMPETITIVA SUSTENTABLE EN UN CLUSTER TURISTICO

\section{Estrategia competitiva}

Propiciar dentro del cluster, un grupo fuerte de rivales competitivos entre si (nacionales e internacionales):

Identificar los actores y métodos con las competencias necesarias paralacreación y entrega de mas valor al cliente (en todo el ciclo del producto de la empresa);

Crearun ambiente local sofisticado que capacita incentiva a las empresas para competirintemacionalmente:Considerarque diversificación tiene mas probabilidadde éxitocuando surge en "capacidades" en los cuales la empresaya compite con éxito Seleccionarsegmentosenloscualesel c/ustertiene oportunidades de ofrecer ventajascompetitivassustentables;

Permitiratenderlos mercados donde compradoressofisticados radican;

Determinar las actividadesenla cadenade valor que deben ser dispersas por los paises que ofrezcan mayores ventajas (sourcing global, al mismo tiempo en quebusca mejorarl los abastecedoreslocales. La mejor formade fidelidad alos abastecedores locales,
es manifestarles, en témninos claros, la necesidadde equipararse, a los extranjeros, en calidad y productividad, a fin de mantenere negocio;

Proporcionara las empresas anexas del ciclo del producto, la información yel acceso a toda innovacióno trabajo científico que está siendo realizado en el mundo y que se relacione conlaindustria; Desarrollar las alianzas estratégicas para obtenereconomías de escala, obtener acceso a la tecnología y los mercados, conseguir

Buscarpadrones ISO, certificaciónde la sustentabilidady etiquetas ecológicas.

Estrategia cooperativa

erun valor superior; Definir y promovertos objetivos delcluster entrelos elementus y actores. Lacoordinaciónde las condiciones parapoderactuardebe
ser definida por los objettivos y marco legal del cluster;

Desenvolvery compartir los valores de la imagen del clusterentre los actores;

Estimular un clima de confianza, compromiso y cooperaciónentre los actores;

Desenvolverla función integradora externae intema, trayendolas necesidades delmercado para dentro del cluster, integrandosus miembros y recursos en la atención de las necesidades de largoplazo de sus clientes;

Crearunared de relaciones cuyo objetivo es desarrollo de la competitividaden todalacadena. Entregarvalor almercado por medio de relacionamientos cooperativos entre empresas independientes en todo el ciclo turistico:

Fomentar, facilitar eincentivar: inversiones públicas/privadas, investigación cientifica, I+D, asociaciones de clase;

Planear, ejecutary disponerun banco de informaciones para todos los agentes. Proyectarde forma compartidalas estrategias de marketing. Construirestratégicame

\section{Estrategia de relacionamiento con el cliente}

Crear, entregary mantener beneficios tangibles para elmejor relacionamiento con el cliente en el ciclo turistico:

Enfatizar principalmente la necesidad de relacionamiento a largo plazo con el mercado, en detrimento de las prácticas de Iransacciones con objetivos de corto plazo;

Analizar y desarrollar mejores politicas habilidad superior en relacionarse con el mercado;

Proporcionar bancos de datos que cuantifique y cualifique las necesidades de los clientes a lo largo de su ciclo;

Solidificar relacionamientos, transformar clientes indiferentes en leales, la calidad de los servicios es lo que debe inspirar a las actividades de marketing (lo importante es la lealtad del cliente; la conquista de nuevos clientes es intermediaria en e
proceso de marketing):

Aplicar el marketing de relacionamiento en toda la cadena de valor de la empresa, abarcando todos los relacionamientos durante el ciclo de elaboración integrado del producto turistico. Incluye región emisora.

Fuente: los autores

\section{Caso: Costa Rica}

El cluster de turismo de Costa Rica se encuentra en desarrollo y en su experiencia práctica ha tenido que resolver situaciones riesgosas para su sobre vivencia (Segura, Inman, 1998). La ventaja comparativa más importante de este cluster, es la diversidad de atractivos turísticos naturales concentrados geográficamente, que sumados sinérgicamente con las actividades turísticas desarrolladas por los agentes, perfeccionan su imagen como destino de turismo ecológico. Tiene, al igual que los otros destinos de la región, la ventaja de fácil acceso desde su mercado más importante (América del Norte) y accesos relativamente fáciles desde otros importantes mercados emisores (especialmente Europa).

La perspectiva de sus empresarios y de las organizaciones públicas, agrupadas cooperativamente, tienen el poder de convertirla en una ventaja competitiva en el ámbito mundial. El cluster enfoca así su energía integradora en perfeccionar los reconocidos atractivos turísticos (comunes a toda la región), con sus valores específicos diferenciados, como son su democracia, estabilidad política, paz social, organizaciones públicas-privadas eficientes, cultura ecológica, sistema de salud y seguridad.

Este cluster tiene una estrategia de marketing adecuadamente planeada, basada en dos factores fundamentales:

a) la competitividad de las propias empresas y sus interacciones, enfocada al objetivo de poder cubrir y especializarse en diferentes nichos del mercado turístico mundial;

b) colocar eficientemente la oferta en "mercados objetivos", disponible para grupos con intereses, necesidades y expectativas particulares (ejemplo: tercera edad, salud, estudiantes, naturalistas)

Estos dos factores han permitido crecer la participación del cluster en el

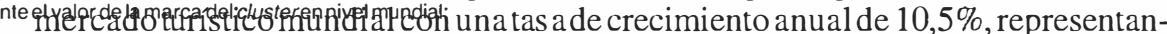
do una importante fuente de empleo directo eindirecto (OMT, 1998). Esta estrategia es desarrollada por un conjunto de empresas diversificadas e intersectoriales victoriosas y competitivas en el ámbito mundial, consiguiendo ofrecer al consumidor final un producto integrado de calidad que satisfaga sus expectativas y necesidades.

Como ejemplo, el departamento de Recursos Naturales del Instituto Costarricense de Turismo (ICT, 1999) ha elaborado el programa de Certificación de la Sustentabilidad Turística(CST), como estrategia para transformar el concepto de sustentabilidad en algoreal, práctico y necesario en el contex to de la competitividad turística del cluster.

Costa Rica presenta la capacidad de realizar innovaciones principalmente en el segmento de turismo ecológico; esta capacidad es reconocida mundialmente por el hecho que, al mismo tiempo en que es capaz de explotarlo sustentablemente, se ha convertido en unos de los principales destinos ecológicos del mundo. El hecho de 
explotar sustentablemente, de forma competitiva, sus recursos naturales turísticos, revela que tanto en la teoría como en la práctica de un cluster turístico, es viable el tratamiento del binomio competitividad-sustentabilidad.

En el análisis de este cluster, en la actual etapa, se destaca que está en el proceso proactivo de aprender el "idioma" del mercado turístico globalizado y de su turista "perfil ecológico" a través de una oferta altamente concentrada y especifica. La capacidad de ser promovida mundialmente se basa en la articulación de una fuerte estrategia cooperativa y de relacionamiento con su turista.

\section{Caso: Nordeste Brasileño}

El gobierno Federal de Brasil y el BID buscan promover una nueva cultura para el desarrollo de la región a partir de la formación de clusters turísticos.

Según The Pro-Northeast Initiative (1999), la región Nordeste está contemplando la segunda fase de la organización de sus clusters de turismo. Durante la primera fase fue desarrollado un diagnóstico inicial con la evaluación de datos nacionales e internacionales con más de 100 entrevistas a los representantes de los polos turísticos locales y de los Estados. Subsecuentemente, fueron organizados grupos participativos de trabajo en tres estados de la región Nordeste, los cuales iniciaron un proceso de identificación de los desafíos competitivos y soluciones compartidas.

La OMT (1998) señala que el Nordeste es actualmente un mercado de servicios regional, con una creciente tasa de turistas nacionales, reflejado por los 2,95 millones de visitantes domésticos y 615 mil visitantes internacionales en 1998. Del 80\% de las visitas nacionales 1, 685 millones, o sea, el 55\%, son de las regiones vecinas al Nordeste. Tiene a Argentina y América del Norte como los principales mercados extranjeros de visitantes a la región. Estos flujos internacionales varían para cada Estado.

Se estudiaron los países competidores claves de turismo a escala mundial, as como, los clusters turísticos de la región. En este proceso de diagnóstico del mercado se seleccionaron cuatro competidores referenciales principales. Estos clusters fueron escogidos por ser competitivos en el ámbito mundial, por presentar en su gestión "las mejores prácticas" y, principalmente, porque compiten en los mismos mercadosque los clusters del Nordeste proyectan posicionarse. Los clusters evaluados fueron Cancún, Bahamas, Costa Rica y República Dominicana. Varios estudios e análisis se realizaron sobre la base de los indicadores; funcionalidad y posicionamientos de los clusters caribeños (The Pro-Northeast Initiative, 1999). Los promotores de los clusters del nordeste se han propuesto las siguientes metas estratégicas:

a) definir su imagen de marca en el ámbito mundial;

b) desarrollar, diversificar e integrar sus productos turísticos; c) actualizar las instalaciones y la capacitación del personal;

d) planificar colectivamente la infraestructura para el turismo.

Según The Pro-Northeast Initiative (1999) el mayor desafío que el Nordeste enfrenta, en el desarrollo de sus clusters de turismo, no radica en la falta de conocimiento interno sobre sus problemas, y sí, en la voluntad y capacidad de las fuerzas regionales de asumir la responsabilidad y trabajar sinérgicamente para resolverlos.

El resultado del análisis realizado con el modelo SIIC, sobre el desarrollo de losclusters del Nordeste, muestra que ésta, está en una fase incipiente de desarrollo. Esta fase puede ser considerada como de estructuración, al estilo de polos turísticos, donde están siendo trabajados principalmente los elementos de la masa crítica y naturaleza de la demanda. Dado el actual estadio de desarrollo del cluster, han sido poco desarrolladas las dimensiones estratégicas para alcanzar la ventaja competitiva de un cluster (competitivas, cooperativas y de relacionamiento).

\section{Consideraciones Finales}

Se aplicó la metodología del SIIC para poder recoger las primeras experiencias de desarrollo de la configuración en clusters turísticos, determinándose que la actividad relevante que diferencia un cluster turístico de éxito, de otro tipo de agrupación organizacional es la integración de la estrategia competitiva con la cooperativa para la satisfacción de las expectativas de sus clientes. Se resume en el Cuadro 2 las actividades del resultado metodológico al aplicar el modelo SIIC.

Se destaca que polos turísticos actuales trabajan principalmente los elementos delSIIC, como son la masa crítica y naturaleza de la demanda. Para su desarrollo y éxito en la globalización debe ocurrir un cambio en sus perspectivas estratégicas, mediante la elaboración integrada de estrategias competitivas, cooperativas y de relacionamiento con el cliente. Para los clusters en desarrollo, es preciso, en la actual fase de la globalización, trabajar la capacidad de las estrategias: competitiva, cooperativas y de relacionamiento con el cliente para poder desarrollar la sustentabilidad de la ventaja competitiva del cluster.

Este tipo de configuración turística en, cluster, al ser analizada por el SIIC presenta que la sensibilidad de sus acciones y de sus vínculos directos están comprometidos con el medio ambiente y la población regional. Señala que la actividad turística tiene mucho que ofrecer y que ganar al comprometerse con el desarrollo sustentable regional. Esto es particularmente significativo en los países menos desarrollados, porque la actividad turística debidamente planificada en cluster, constituye tanto un sostén del desarrollo, como potencializador del flujo de redes de inversiones, conocimiento y tecnologías entre otros. 
CUADRO 2 - ANÁLISIS DE LAS ACTIVIDADES DE UN POLO Y UN CLUSTER TURÍSTICO SEGÚN EL MODELO SIIC

\begin{tabular}{|c|c|c|}
\hline \multirow{2}{*}{ 4ctrododosaroladr. } & \multicolumn{2}{|c|}{ Throdeconiguracion } \\
\hline & rool & culuster. \\
\hline Oferta & Dispersa & $\begin{array}{l}\text { Segmentos especinioos. Integ rada para } \\
\text { satisfacer expectativas de los turi stas. } \\
\text { Productos planificados e integrados }\end{array}$ \\
\hline Demand a & No caificada & Caificada, cientes sofisticados \\
\hline Competidores & Depredatorios, sin leyes & Maroo legal detalado e incentivador \\
\hline Tecrobgia & $\begin{array}{l}\text { ndividual Orienta da al } \\
\text { proceso }\end{array}$ & $\begin{array}{l}\text { Elaboración, uso y distribución } \\
\text { compartida por los agentes. Orientada al } \\
\text { diente }\end{array}$ \\
\hline Estrategia Competitiva & Destructiva & $\begin{array}{l}\text { Fuerte, constructiva, alianzas estraté gicas } \\
\text { a escala regional y mundial }\end{array}$ \\
\hline Estrategia Cooperativa & No existe & $\begin{array}{l}\text { Planificada e inte grada al cliente. } \\
\text { hiciativa píb lica, privada y no } \\
\text { gubernamental. }\end{array}$ \\
\hline $\begin{array}{l}\text { Estrategia de } \\
\text { relacionamientos con el } \\
\text { turista }\end{array}$ & $\begin{array}{l}\text { No existe, o individual por } \\
\text { algunos agentes }\end{array}$ & $\begin{array}{l}\text { Integrada para el cluster. Planificada para } \\
\text { cada agente y sociedad local }\end{array}$ \\
\hline $\begin{array}{l}\text { Sustentabilidad del } \\
\text { desarrollo }\end{array}$ & No existe & Altamente planificada y reglamentada \\
\hline $\begin{array}{l}\text { Relación de los sectores } \\
\text { público, privado y no } \\
\text { gubernamental }\end{array}$ & Pooos agentes to realizan & $\begin{array}{l}\text { Es obligatorio para el clustery para } \\
\text { algunos agentes }\end{array}$ \\
\hline Imagen & De cada agente & $\begin{array}{l}\text { Del cluster en conjunto. Regional y } \\
\text { mundial }\end{array}$ \\
\hline $\begin{array}{l}\text { Ciclo de la actividad } \\
\text { turistica }\end{array}$ & Fragmentada & Productos integrad os y diversificados \\
\hline Diagnósticos y consultorias & hdividual & En $\infty$ rijurto e individual. Sazonal. \\
\hline $\begin{array}{l}\text { Definición de las políticas } \\
\text { de acción y maroo legal. }\end{array}$ & No hay & $\begin{array}{l}\text { ndispensable. Empresas y gobiemo } \\
\text { alineados en los objetivos. }\end{array}$ \\
\hline $\begin{array}{l}\text { Estrategia para el } \\
\text { desarrolb. }\end{array}$ & A corto plazo e individual & A argo plazo, en conjurto e individual. \\
\hline
\end{tabular}

\begin{tabular}{|c|c|c|}
\hline \multirow{2}{*}{ Actridad o s s anolladd } & \multicolumn{2}{|c|}{ Tporocorigurecior } \\
\hline & robo. & chinster \\
\hline Planificación estratégica. & & $\begin{array}{l}\text { Empresas y cluster con objetivos } \\
\text { mindiales. }\end{array}$ \\
\hline $\begin{array}{l}\text { Educación, Capacitación y } \\
\text { Cultura }\end{array}$ & Alginos individualmerte & En $\infty$ rijunto e individual \\
\hline Ejecución y Promoción & ndividual & En $\infty$ rijunto e individual \\
\hline $\begin{array}{l}\text { Evaluación y } \\
\text { retroalimentración }\end{array}$ & No hay & Detalada y obligatoria \\
\hline $\begin{array}{l}\text { Certificación de la } \\
\text { sustentabilidad }\end{array}$ & No hay & Se exige \\
\hline Etiq Letas eoológicas & Indiferente & $\begin{array}{l}\text { ncentivos en el ámbito de clustery de } \\
\text { empresas }\end{array}$ \\
\hline Agencias en el exterior & Embajada del país & $\begin{array}{l}\text { Agencias y articulaciones propias del } \\
\text { cluster }\end{array}$ \\
\hline Investigación, I+D & ndividual & En $\infty$ rjunto e individual \\
\hline Participación Social & No hay & Exigida y planificada \\
\hline $\begin{array}{l}\text { Fomento e incentivo a } \\
\text { inversiones nacionales y } \\
\text { extranjeras }\end{array}$ & hdividuales & $\begin{array}{l}\text { Altamente planificada y articula da en todo } \\
\text { el mundo }\end{array}$ \\
\hline $\begin{array}{l}\text { Enfoque y planificación } \\
\text { intersectorial }\end{array}$ & No existe & $\begin{array}{l}\text { Se trabaja œn meto dologia y técnicos } \\
\text { especificos ( ho rizontal y verticalmente). }\end{array}$ \\
\hline
\end{tabular}

\section{Bibliografía}

ACHROL, R. 1991. Evolution of the marketing organization: new forms for turbulent environments. Journal of Marketing, $v 55$, oct

BENI, M. 2000. Análise Estrutural do Turismo. São Paulo: SENAC.

CRAVENS, D.; PIERCY, N. 1994. Relationships marketing and cnlaborative network in service organization

International Journal of Services Industry Management, v.S, n.5.
DULL, D.; MOHN, L.: NOREN, E. 1995. Parteners. The McKinsey Quarterly, n. 4, Dec. 2.
GUTIÉRREZ E. BORDAS, E. 1993. La competitividad de los destinos turisticos en mercados lejanos. AIEST, Suiça: GUTIÉRREZ, E; BORDAS, E. 1993. La competitividad de los destinos turisticos en mercados lejanos. AIEST, Suif̧ St. Gallen.

HUNT, M.; MORGAN, R. Relationships marketing in the era of networkcompetition. Marketing Management, v.3, n. 1 .

MANNING, E 1993. Managing Sustainable Tourism: Indicators for Better Decisions, Proceedings, 1992 World 
Congress on Adventure Travel and Ecoturism, Canadá: Whistler.

MCKENNA, R. 1993. Marketing de Relacionamento: estratégias bem-sucedidas para a era do cliente. Rio de Janeiro: Campus.

MINTZBERG, H.; QUINN, J. 1998. O processo da estratégia. Porto Alegre: Bookman.

ORGANIZACIÓN MUNDLAL DEL TURISMO. 1998. Compendio de Estadística. Madrid.

PORTER, M. 1998. Clusters and the new economics of competition. Harvard Business Review, november, december. 1996. A vantagem competitiva das naçôes. Rio de Janeiro: Campus.

SEGURA, G.; INMAN C. Turismo en Costa Rica: la visión a largo plazo. Costa Rica: CLACDS.

SLATER, F.; NARVER, J. 1995. Market orientation and leaming corganization. Journal of Marketing, v. 59, July.

SMITH, S. et al. 1999. South Australian International Tourism Industry Cluster. Background Paper, Report Meeting \#1

February, Report Meeting \#2 April, Canberra.

THE PRO-NORTHEAST INITIATIVE. 199. Relatório Técnico sobre Turismo Phase II Report. Bahia: Bahiatur.

TOLEDO et al. 2001. Marketing y Competitividad de Empresas Turísticas en Ambiente Globalizado. Estudio de casos de clusters turísticos. Anais XXXVI CLADEA, México.

VALLS, J. 1996. Las claves del Mercado Turístico. Bilbao: Deusto.

WEBSTER, F. 1992. The changing role of marketing in the corporation. Journal of Marketing, v 56, Oct.

Recibido en 4/2/2002

Aprobado en 13/2/2002 